\title{
Erratum: Instantons from blow-up
}

Joonho Kim, ${ }^{a, 1}$ Sung-Soo Kim, ${ }^{b}$ Ki-Hong Lee, ${ }^{c, 2}$ Kimyeong Lee $^{a, 1}$ and Jaewon Song ${ }^{a, 1}$

${ }^{a}$ Department of Physics and Astronomy \& Center for Theoretical Physics, Seoul National University,

1 Gwanak-ro, Gwanak-gu, Seoul 08826, Korea

${ }^{b}$ School of Physics, University of Electronic Science and Technology of China, No. 4, section 2, North Jianshe Road, Chengdu, Sichuan 610054, China

${ }^{c}$ School of Physics, Korea Institute for Advanced Study, 85 Hoegiro, Dongdaemun-gu, Seoul 02455, Korea

E-mail: joonhokim@kias.re.kr, sungsoo.kim@uestc.edu.cn, khlee11812@gmail.com, klee@kias.re.kr, jsong@kias.re.kr

ERRATUM TO: JHEP11(2019)092

ARXIV EPRINT: 1908.11276

Corrected the authors' affiliations. Affiliation a and c were swapped.

Open Access. This article is distributed under the terms of the Creative Commons Attribution License (CC-BY 4.0), which permits any use, distribution and reproduction in any medium, provided the original author(s) and source are credited.

\footnotetext{
${ }^{1}$ Correct affiliation is c: School of Physics, Korea Institute for Advanced Study, 85 Hoegiro, Dongdaemungu, Seoul 02455, Korea.

${ }^{2}$ Correct affiliation is a: Department of Physics and Astronomy \& Center for Theoretical Physics, Seoul National University, 1 Gwanak-ro, Gwanak-gu, Seoul 08826, Korea.
} 